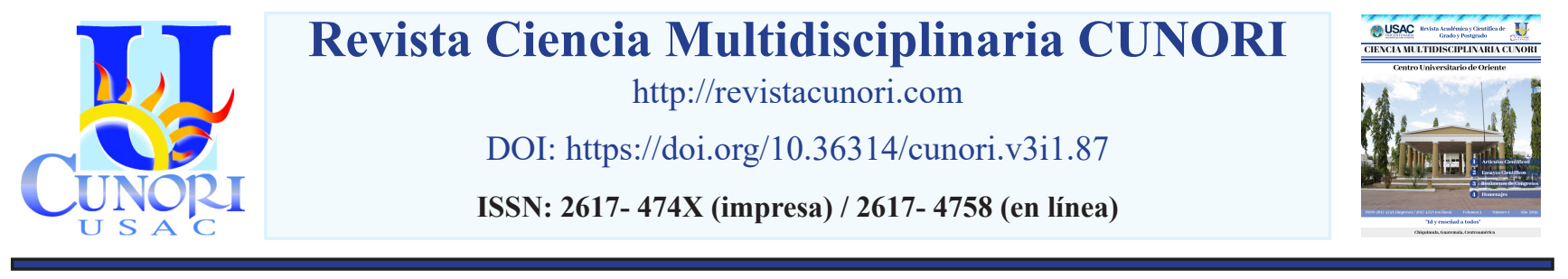

Como citar el artículo

Ramírez, L. (2019). Modelo para la integración de salud y sexualidad en el desarrollo curricular. Revista Ciencia Multidisciplinaria CUNORI. 3(1). 117-125. DOI: https://doi.org/10.36314/cunori.v3i1.87

\title{
Modelo para la integración de salud y sexualidad en el desarrollo curricular
}

\section{Model for the integration of health and sexuality in curricular development}

\author{
Lilian Carolina Ramírez Castellanos* \\ Facultad de Humanidades, Universidad de San Carlos de Guatemala \\ Recibido: 17 de diciembre de 2018 / Revisión: 25 de enero de 2019 / Aceptado: 12 de febrero de 2019
}

Disponible en internet el 30 de agosto de 2019

Resumén

*Autor para correspondencia.

T a educación en salud y sexualidad es importante porque es fundamental para la vida y desarrollo humano, sin embargo, es poco Lusual que personas jóvenes reciben educación sexual que llene condiciones apropiadas para la vida y que incluya elementos culturales apropiados. La investigación que se expone en este artículo es descriptiva y propositiva. El entono escolar, es apropiado para entregar educación sexual antes del inicio de la vida sexual activa y dentro del marco de la prevención y mucho que mejor que hacerlo a través de una estructura curricular adecuada. El desarrollo de un modelo curricular además de los contenidos teórico académicos, debería contribuir a responder a las principales necesidades y prevención de problemas de la sociedad en la que se implementa, en este aspecto, esta propuesta de modelo contribuye a responder a la preocupación planteada por la Organización Mundial de la Salud, para el período de adolescencia, justo cuando se proyecta el futuro de personas individuales, dentro de la sociedad, con proyección hacia el futuro, principalmente como herramienta para la prevención de uno de los principales problemas sociales en Guatemala como es el embarazo en adolescentes, que a su vez está relacionado con menor rendimiento académico, dificultades en el mercado laboral y desarrollo social de las mujeres con el consecuente impacto para la sociedad Guatemalteca.

Palabras clave: modelo curricular, adolescentes, educación en salud y sexualidad, embarazo en adolescentes

\section{Abstract}

T is important the education about heath and sexuality because it is fundamental to define the future of the life and to improve the Lhuman development. However, it is unusual that the young people have receive health education and reproductive and sexual education for their life taking in count the cultural and social elements to achieve suitable conditions for their life. This research work, make reference to the descriptive and purposeful research. The scholar environment is an appropriate frame of work to offer sexual education, before that the active sexual life be started, and inside de framework of the prevention and better done through a suitable defined curricular structure. In addition to the academic and theoretical contents, the design of a curricular model, must be contribute to answer to the main needs to prevent the social problems. This proposal using a model that contribute to answer the great concern indicated by the World Health Organization, regarding the adolescence period, that is the same point of the time where the young people is designing the future of their own life within the society, with a future projection, mainly as a tool to prevent one of the main social problems to the Guatemalan society, as the teen pregnancy, which is turn associated with another kind of problems to impact with the social development, like the poor academic performance and difficulties to reach the labor market.

Keywords: curricular model, adolescents, education in health and sexuality, pregnancy in adolescents 


\section{Introducción}

El grupo de adolescentes constituye un grupo de población con necesidades especiales, el 95\% de los niños nacidos de madres adolescentes provienen de países en desarrollo por lo que existe interés en buscar y desarrollar intervenciones específicas para la prevención del embarazo en adolescentes, la Organización Mundial de la Salud ha indicado que es importante suministrar información que se incluya el tema de sexualidad en la educación para la salud así como el desarrollo de habilidades, buscar retener a las adolescentes tanto en la primaria como en secundaria, ofrecer intervenciones que integren y combinen programas de educación sexual para mejorar oportunidades a través de canales formales y no formales (Organización Mundial de la Salud, 2011 : 4, 145, 160), así mismo diseñar intervenciones con enfoque adaptado a sus realidades e involucrar y facultar a la población de jóvenes para que participe en el diseño, aplicación y seguimiento de las intervenciones estratégicas (Organización Panamericana de la Salud, $2018: 34)$.

\section{Materiales y métodos}

\section{Importancia de la integración de salud y sexualidad en el desarrollo curricular}

El desarrollo de este modelo, contribuye a responder a la preocupación planteada por Organización Mundial de la Salud, considerando que el período de adolescencia que va de los 10 a 19 años es una etapa en la que se proyecta el futuro de las personas individuales, dentro de la sociedad con proyección hacia el futuro, también es un momento en el que se puede aprovechar la oportunidad para generar cambios (Organización de las Naciones Unidas para la Educación la ciencia y la Cultura, 2010: 2-7). Por otro la prevención de embarazo en adolescentes compromete una reducción de años de escolaridad, disminuyendo la asistencia y número de horas de trabajo (Banco Mundial, 2012:17). El rol de la escuela y de los padres en la protección de las y los adolescentes en contra de comportamientos que los exponen a riesgo, como lo son las prácticas sexuales es fundamental para alcanzar que se cumplan los objetivos de disminución de riesgos de embarazos en este grupo de edad (Organización Mundial de la Salud, 2014:12).

El modelo curricular de Ralph Tyler (1973) ha sido históricamente determinante en el diseño curricular y ha influido de forma muy determinante desde el siglo pasado e inicio del siglo veintiuno y constituyó la base teórica para esta propuesta (Tyler, 1973).El modelo de Tyler sigue vigente en el diseño curricular (Vélez, Laura, \& Delgado, 2010:57) y se espera que pueda ser utilizado por docentes o educadores responsables de impartir educación en el nivel de formación principalmente a nivel primario. Dadas las condiciones precedentes es necesario contar con un modelo curricular para que cualquier docente de nivel primario pueda diseñar el curso de educación integral en salud y sexualidad para niñas y niños adolescentes y aplicarlo en escuelas de la ciudad de Guatemala.

\section{Descripción y aplicación operativa del modelo}

El modelo de Tyler se enfoca en el contexto social y con un enfoque en el alumno se propone para que pueda ser utilizado en la implementación por cualquier docente o educador a nivel micro, para la implementación de currículo y contenidos en educación integral en salud y sexualidad con condiciones apropiadas para la vida, con elementos culturales apropiados, dentro el entono escolar. Esta propuesta surgió a partir del diseño curricular descrito por Tyler y se fundamentó en cada uno de los principales pasos 
y puntos que se desarrollan por el autor (Tyler, 1973). Es necesario tener claro que los intereses identificados en el estudiante constituyan el comienzo para la enseñanza efectiva. Además es indispensable tener acceso a examinar datos de salud, de la/s comunidad/es a las que se propone alcanzar, analizar estadísticas de morbilidad y mortalidad relacionadas con el problema de la ocurrencia del embarazo en adolescentes, revisar cualquier documentación de investigaciones relacionadas con indicadores de salud de la población a la que se enfocará.

\section{Paso 1. La identificación de necesidades}

Se propone identificar las necesidades de los y las estudiantes a través de investigación con enfoque de teoría progresiva, para que el interés de los estudiantes sirva como centro de atención de quienes serán los educadores (Tyler, 1973:17). Al mismo tiempo la investigación deberá ser enfocada en normas deseables relacionadas con aspectos sociales, culturales, familiares, relaciones cívicas y otras características que sean consideradas importantes en el contexto en el que se desenvuelven los y las estudiantes. Todo esto para que sea la base para definir los objetivos, por lo tanto, tendremos objetivos circunscritos al contexto y circunscritos a los intereses del estudiante (cuadro 1).

\begin{tabular}{llcll}
\hline Paso & Estado actual & Necesidades & Norma deseable & \\
Carencias o vacíos & & Salud & \\
& "Teoría progresiva" & & Vida familiar & Punto Base \\
para comparar & Vida Social & cambios en la \\
evaluación
\end{tabular}

Cuadro 1. Paso uno -Identificación de necesidades para definición de modelo educativo.

Fuente: Elaboración propia a partir del modelo de Tyler (Tyler, 1973) 
Se espera que los intereses identificados en el estudiante constituyan tal como lo indica el libro de Tyler el comienzo para la enseñanza efectiva (Tyler, 1973 :17)

Así mismo la información respecto a la vida contemporánea de las y los estudiantes a los que se dirigirán los objetivos (Tyler, 1973 : 21). En este sentido tener acceso y examinar datos de salud, de la/s comunidad/es, analizar estadísticas de morbilidad y mortalidad relacionadas con el embarazo en adolescentes, revisar cualquier documentación de investigaciones relacionadas con indicadores de salud de la población a la que se enfocará.

A partir de allí, realizar investigación previa y documental, relacionada con los temas de salud, vida familiar, vida social, relaciones cívicas, vida profesional y otras que sean considerada de alta importancia que surjan durante el proceso de documentación y dependiendo de categorías que sean identificadas (Tyler, 1973:15).

Paso 1.1 Aplicado a la temática, la Investigación de fuentes para poder definir los objetivos, esto deberá ser realizado a través de:

A. Identificar los intereses del estudiante: para que los resultados sirvan como centro de atención de los educadores, de manera que deberán ser consideradas todas las circunstancias de contexto y circunstancias que interesan al estudiante y que están relacionadas con la educación integral en salud y sexualidad, el implementador deberá tener un nivel de conocimiento claro respecto a cuales son los fines de los objetivos que se espera construir en términos de prevención de embarazo en adolescentes y prevención de la deserción escolar.

B. Realizar investigación de la vida contemporánea fuera de la escuela y que esté relacionada con el contexto de la población local al momento de la investigación, de manera que deben ser descritos los elementos que de una u otra forman parte del espacio donde se desenvuelve y desarrolla el grupo de población al que está dirigido este modelo en términos de vulnerabilidad. Estos aspectos principales son; sanitarios, familiares, vocacional, religiosa, cívico, entre otros que están directamente relacionados con la ocurrencia del problema.

\section{Examinar con relación a la filosofía de la institución}

Se deberán considerar los elementos filosóficos que dirigen el aprendizaje dentro de la institución principalmente, sin dejar fuera la relación de la filosofía de la escuela con la que es parte del contexto propio de los adolescentes. Los implementadores deben estar en capacidad de realizar investigación en la que el enfoque filosófico sea enfocado en prevención, respeto, género, igualdad, identidad y otro valores filosóficos que den más soporte a la credibilidad del modelo.

\section{El modelo no considera priorizar en función de la psicología educativa}

Hasta este momento el implementador deberá estar en capacidad para responder a dos de las preguntas indicadas por Tyler: ¿Qué fines desea alcanzar la escuela? y ¿Cuáles son las experiencias que ofrecen mayores probabilidades de alcanzar los fines definidos? 
Para ello es necesario que implementador se plantee la pregunta ¿Cómo puede establecer que conocimientos deben adquirirse? Los conocimientos serán determinados en función de los resultados de la investigación inicial, para saber cuál es el conocimiento que deben adquirir los estudiantes de educación primaria, para ello se deberá realizar investigación específica dentro de los centros educativos que se espera abordar. Deberá realizarse paralelamente un proceso de investigación de variables puntales relacionadas con los docentes que formarán parte de la implantación del conocimiento dentro de la institución debido a que permitirá identificar elementos filosóficos y de valores que favorecen o que limitan el tema de prevención de embarazo en adolescentes y educación integral en salud y sexualidad.

El enfoque del modelo será establecido para el nivel primario con detalle de: Destrezas y habilidades y los saberes prácticos se necesitan según sea el nivel de desarrollo al que se aplicará el modelo atendiendo específicamente a los grados que se esté enfocando.

\section{Paso 2. La elaboración y selección de los objetivos}

Deberá ser construido a partir de la base del diagnóstico de necesidades; desde este punto se obtendrá el listado de objetivos sugeridos y priorizados para la selección de los que serán propuestos.

La lista de objetivos importantes y alcanzables deberá contener información del contexto y del interés de los estudiantes, con enfoque de género, prevención, respeto y convivencia así como identidad para fortalecer estos elementos y otros que hayan sido identificados, de manera que se espera que los estudiantes participen voluntariamente, en un ambiente cómodo, acorde a sus necesidades de información y convivencia dentro del que se practique respeto, autoestima, sana convivencia y al mismo tiempo que los implementadores sean capaces de identificar conductas de riesgo en las que se requiera involucrar otros niveles de participación como por ejemplo si se considera apropiada o no la participación de los padres dentro del desarrollo de este proceso educativo institucional, también deberán ser identificados cuáles son esos espacios de participación de los padres y quedar claramente descritos dentro de los objetivos.

Esquema 1. Paso 2 - objetivos base a partir del diagnóstico de necesidades

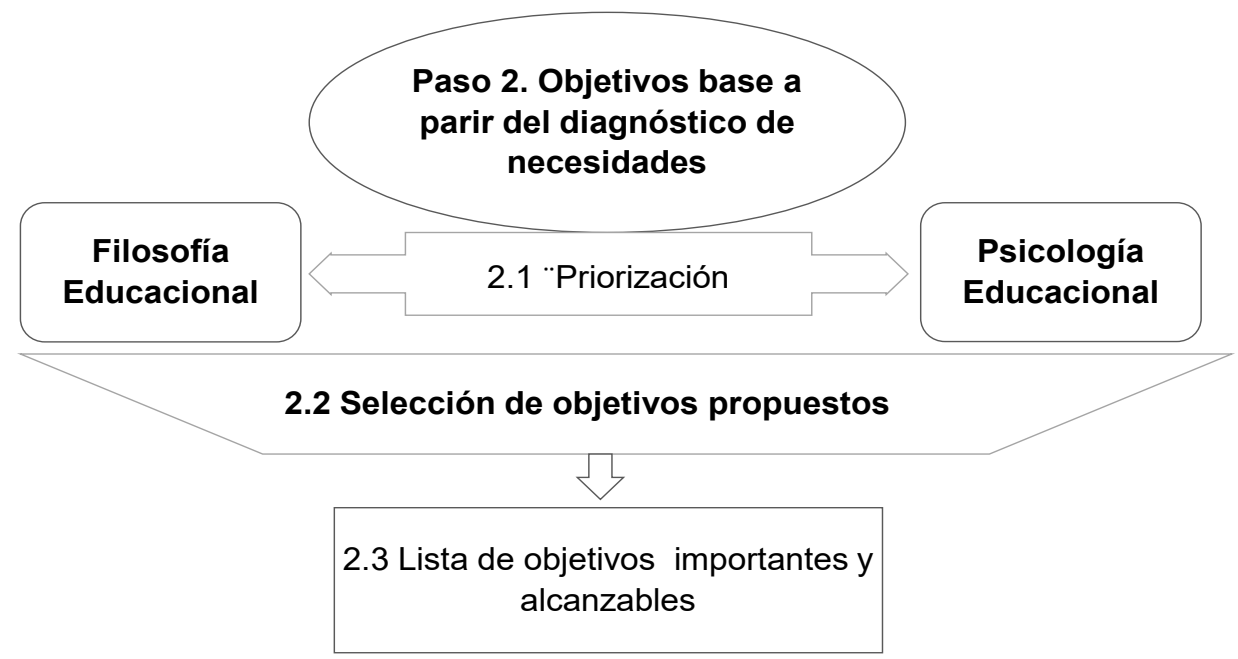

Fuente: Elaboración propia a partir del modelo de Tyler (Tyler, 1973) 


\section{Paso 3. Selección de actividades prioritarias}

La selección de las actividades debe priorizar las que permitan al estudiante confrontar e identificar situaciones de riesgo para prevenir el embarazo en adolescentes y el abandono escolar para que de esta forma se dé al estudiante la oportunidad de ejercitar el pensamiento y estimular la conducta más conveniente (Tyler, 1973:71).

En este caso se proponen los siguientes criterios para definir las actividades: Actividades permitirán al estudiante estar en contacto con elementos de riesgo para la ocurrencia de embarazo así como las consecuencias de que este ocurra durante el período de adolescencia. Actividades que permitan que los estudiantes participen rápidamente de actividades en las que se fortalezcan procesos que ofrecen satisfacción por la participación.

Actividades que permitan al estudiante poder cumplir en la participación durante la mayoría del tiempo a través de considerar que la conducta de los estudiantes debe ser acorde a las condiciones de las actividades que se están proponiendo. Actividades dosificadas de acuerdo a edades con diferenciación de niveles de conocimiento al tema, contexto habilitante a la aceptación del tema, de manera que se obtenga un proceso de actividades de implementación modular con dosificación gradual y prolongada, es decir que durante el mismo período (un año escolar) se dosifiquen las actividades.

Realizar tablas de cotejo que ordenen posibles sinergias entre las actividades de los objetivos, de manera que al final se identifiquen puntos nuevos de sinergia que favorecerán el cumplimiento de los objetivos y el alcance propuesto. Las actividades que finalmente se espera alcanzar deben ser actividades de aprendizaje para actitudes sociales y para producir interés en desarrollar satisfacción. Para la organización de los objetivos se debe ordenar las actividades, cuidando los tres elementos de los principios organizadores indicados en el modelo de Tyler: continuidad, secuencia e integración por cada nivel (Tyler, 1973:99), de manera que no se pierda el objetivo dentro de cada uno de los ciclos en los serán enfocadas las actividades. Con esto se insta a generar una secuencia de manera que se inicie en el nivel primario para que pueda haber continuidad, secuencia e integración por grados y niveles.

Por otro lado también se propone que la estructura organizativa opte por desarrollar la planificación para un lapso prolongado es decir continuidad durante todos los grados de un solo nivel. Para el caso de Guatemala está justificado porque según el sistema educativo nacional como conjunto interrelacionado de procesos y de sujetos, las acciones educativas se deben realizar con base a necesidades e intereses y es función fundamental del sistema educativo estructurar programas en forma gradual y progresiva (Congreso de la República de Guatemala, 1991).

El subsistema escolar de Guatemala se organiza según niveles, ciclos y grados, en donde el nivel de educación primaria incluye de primero a sexto grado, esto ofrece la ventaja de lograr la organización estructural fragmentando el tiempo en unidades específicas con el correspondiente momento de transición y coloca la oportunidad de prevenir desde antes de la ocurrencia del problema para generar el impacto esperado en la prevención de riesgos. 


\begin{tabular}{l}
\hline \multicolumn{1}{c}{ Criterios } \\
\hline $\begin{array}{l}\text { Enfoque de } \\
\text { actividades }\end{array}$ \\
experiencia señalada \\
Que ofrezcan "satisfacción" \\
Que la conducta sea en \\
función de las posibilidades \\
del alumno \\
Actividades orientadas a \\
objetivos \\
Sinergia entre actividades \\
para objetivos \\
Es previsible que la misma \\
actividad requiere que \\
diversos objetivos
\end{tabular}

Cuadro 2. Paso tres - selección de actividades

Fuente: Elaboración propia a partir del modelo de Tyler (Tyler, 1973)

\section{Paso 4. La evaluación}

Debe conservar los mismos criterios de confiabilidad y representatividad aducidos en la medición inicial. Además de considerar los tipos de personas que fueron investigados durante la medición inicial, se considera que en este momento debe realizarse evaluación de la estructura alcanzada respecto al modelo definido, para reconocer el nivel logrado.

La evaluación en función del logro de los objetivos así como la evaluación en función de cambios sujetados a la propia implementación del modelo que se relacionan con conocimiento, conducta y aplicación de actividades después de un período claramente definido que permita observar mayor probabilidad de ocurrencia de cambios. Para la formulación de objetivos, se habrán considerado aspectos de "conducta" a los que se espera llegar y "contenidos" que se requieren para alcanzar los objetivos esperados, mismos que también deben ser evaluados al finalizar el período que sea definido.

\section{Discusión}

El proceso de aprendizaje propuesto como resultado de la aplicación de este modelo, pretende alcanzar que las y los jóvenes adquieran hábitos en salud y sexualidad, así como los conocimientos prácticos, con base a un programa dosificado y continuo de aportes, alineados a edades y características definidas. El proceso debe estar enfocado dentro del marco de la prevención de uno de los principales problemas que enfrenta la sociedad guatemalteca, el embarazo a la edad adolescente cuya consecuencia es nefasta para el progreso y el desarrollo humano, social y económico del país, entre otras consecuencias individuales e inherentes a la mujer. Utilizar una estructura adecuada para el desarrollo de un modelo curricular además de los contenidos teórico académicos, contribuye a responder a las principales necesidades y prevención de problemas de la sociedad en la que se implementa, en este trabajo se propone utilizar un 
modelo que contribuye a responder a la preocupación planteada y que continúa vigente para el desarrollo curricular a pesar del tiempo de su existencia. Realizar propuestas con base a este modelo u otro otorga sustento científico y la estructura organizativa apropiada para generar herramientas participativas, integrales y efectivas a la luz de las necesidades y prioridades de la sociedad a la que sea dirigido. Por otro lado facilita la sistematización de experiencias que podrán ser utilizadas por tomadores de decisiones para futuras intervenciones para la búsqueda de soluciones con participación directa de los propios involucrados en la problemática lo que facilita el éxito de las intervenciones.

\section{Referencias bibliográficas}

Banco Mundial. (2012). Embarazo Adolescente y Oportunidades en América Latina y el Caribe, p.32.

Congreso de la República de Guatemala. (1991). Ley de Educación Nacional. Guatemala.

Organización de las Naciones Unidas para la Educación la ciencia y la Cultura. (2010). Orientaciones Técnicas Internacionales sobre Educación en Sexualidad Un enfoque basado en evidencia orientado a escuelas, docentes y educadores de la salud. Santiago, Chile.

Organización Mundial de la Salud. (2011). Directrices de la OMS para la Prevención del Embarazo Precoz y los Resultados Reproductivos Adversos en Adolescentes de loa países en desarrollo. Retrieved from ISBN 9789143502212

Organización Mundial de la Salud. (2014). Salud para los adolescentes del mundo. Una segunda oportunidad en la segunda década.

Organización Panamericana de la Salud. (2018). Acelerar el progreso hacia la reducción en la adolescencia en América Latina y el Caribe. Washington DC.

Tyler, R. W. (1973). Principios básicos del Currículo. (E. T. S. A., Ed.). Buenos Aires.

Vélez, G., Laura, C., \& Delgado, T. (2010). Modelos para el diseño curricular. Pampedia.

\section{Sobre la autora}

\section{Lilian Carolina Ramírez Castellanos}

Candidata a Doctora por la Universidad de San Carlos de Guatemala, con Maestría en Alimentación y Nutrición en la Facultad de Ciencias Químicas y Farmacia, es Médica y Cirujana, es también estudiante de la Maestría en Gestión y Evaluación de Proyectos en la misma universidad. Becaria de "Tecnología de Investigación en Planificación Familiar (Programa de Entrenamiento en Investigación en Family Health International y Universidad de Carolina del Norte en Carolina del Norte). Ha realizado las siguientes investigaciones: "Factores de riesgo de embarazo en adolescentes y prácticas educativas para la prevención del abandono escolar" y "Modelo para la integración de salud y sexualidad en el desarrollo curricular". 


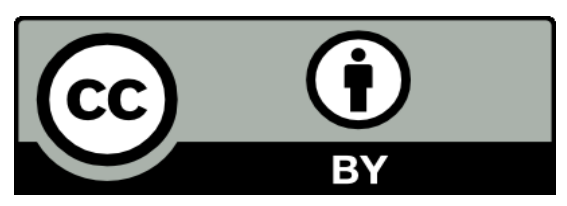

Este texto está protegido por una licencia CreativeCommons 4.0.

Usted es libre para compartir, copiar y redistribuir el material en cualquier medio o formato y adaptar el documento, remezclar, transformar y crear a partir del material para cualquier propósito, incluso comercialmente, siempre que cumpla la condición de atribución: usted debe reconocer el crédito de una obra de manera adecuada, proporcionar un enlace a la licencia, e indicar si se han realizado cambios. Puede hacerlo en cualquier forma razonable, pero no de forma tal que sugiera que tiene el apoyo del licenciante o lo recibe por el uso que hace. 